\title{
Nontuberculous mycobacteria in non-HIV patients: epidemiology, treatment and response
}

\author{
M.T. Henry*, L. Inamdar*, D. O'Riordain*, M. Schweiger", J.P. Watson*
}

Nontuberculous mycobacteria in non-HIV patients: epidemiology, treatment and response. M.T. Henry, L. Inamdar, D. O'Riordain, M. Schweiger, J.P. Watson. (C) ERS Journals Ltd 2004.

ABSTRACT: Recent international guidelines published in 1997 and 1999 have proposed diagnostic and treatment criteria for disease caused by nontuberculous mycobacteria (NTM).

In this paper, the epidemiological data, diagnostic criteria, treatment regimens and outcomes from $117 \mathrm{HIV}$-negative patients who had a positive culture for NTM between 1995-1999 are reviewed. The authors wished to identify factors associated with improved outcome in these patients.

A total of 71 patients were believed to have a clinical disease caused by NTM, as defined by international criteria. A total of $72 \%$ patients were found to have had pulmonary disease. There was a rise in infections between 1995-1999, with a peak in infections in 1997. The most striking rise was in Mycobacterium avium intracellulare complex infections (1995: 33\% infections; 1996: 36\% infections; 1997: 41\% infections; 1998: 61\% infections; 1999: 57\% infections). There was a link between deprivation and number of positive NTM isolates $(34.4 \%$ isolates occurred in the areas of lowest Carstairs deprivation index versus $\mathbf{2 0 . 6} \%$ isolates from areas of least deprivation). There was a significant association between appropriate therapy, defined by American Thoracic Society and British Thoracic Society guidelines, and successful outcome $(74 \%)$ in contrast to those who received inappropriate treatment prior to the publication of these guidelines.

Nontuberculous mycobacteria infections remain a significant problem in non-HIV patients. Adherence to published guidelines may improve patient outcomes.

Eur Respir J 2004; 23: 741-746.
*Dept of Respiratory Medicine, Leeds General Infirmary, and ${ }^{\#}$ Dept of Communicable Disease Control, Leeds Health Authority, Leeds, UK.

Correspondence: M.T. Henry

Dept of Respiratory Medicine

Leeds General Infirmary

Leeds LS1 3EX

UK

Fax: 441133926316

E-mail: michael.henry@leedsth.nhs.uk

Keywords: Epidemiology nontuberculous mycobacterium response-to-treatment guidelines

Received: October 122003

Accepted after revision: January 232004
Although nontuberculous mycobacteria (NTM) were observed soon after Koch's discovery of Mycobacterium tuberculosis, the clinical significance of this group of NTM was not appreciated until the 1950s when they were classified as "atypical mycobacteria," on the basis of their in vitro characteristics. Since this time many large series of both HIVpositive and -negative patients, suffering from both pulmonary and extrapulmonary diseases attributable to what are now described as "nontuberculous mycobacteria," have been described [1-6]. NTM and the pulmonary diseases they cause remain a challenge for microbiologists and pulmonary physicians.

The increase in HIV prevalence in developed countries has contributed to a rise in NTM infections. However, increasing vigilance and awareness of these bacteria as human pathogens, improvements in methods of detection and culture, and increasing contact between humans and NTM have also contributed to the apparent increase in disease burden [7]. There is also evidence that the prevalence of NTM infections is increasing, particularly though not exclusively, in non-HIV patients with underlying lung disease [5, 8-11]. In response to this increase in the burden of disease, the American Thoracic Society (ATS) and the British Thoracic Society (BTS) have issued guidelines on the diagnosis and management of NTM infections [12, 13]. Both sets of guidelines highlight the difficulty in distinguishing patients with clinical disease related to NTM from those in whom the isolation of single clinical specimens raises a clinical suspicion of disease. Both bodies suggest similar therapeutic regimens for individual NTM, in both pulmonary and extrapulmonary disease. Success rates with these regimens are often poor, with failure to eradicate the organisms or recurrence of disease after cessation of therapy. The incidence of NTM infections in nonHIV patients in a large urban centre in the UK, over a 5-yr period, was reviewed by the present authors. Epidemiological factors associated with disease and factors associated with recurrence of clinical infection were also reviewed. Diagnostic criteria and treatment regimens used by clinicians prior to publication of evidence-based guidelines were scrutinised. Subsets of patients whose treatment regimens were consistent with subsequent guidelines were compared with those patients whose treatment regimens were not consistent with the guidelines to evaluate the likely benefit of adherence to the ATS and BTS guidelines on treatment of NTM disease.

\section{Methods}

\section{Subjects and design of the study}

The present authors reviewed the case records of 117 HIVnegative patients coded as suffering from NTM, atypical mycobacteria or opportunist mycobacteria in Leeds, a large 
urban community in the UK with a population of 715,000 including 35,000 (4.8\%) from ethnic minorities born outside the European Union (EU) and 12,000 (1.7\%) born outside the UK but within the EU [14]. A standard proforma was used to extract information from case notes including age, sex, occupation, background respiratory disease status, Bacille Calmette-Guérin (BCG) status, and conditions other than HIV which were likely to impair immune defences, e.g. diabetes mellitus, leukaemia, lymphoma, rheumatoid arthritis, corticosteroid and immunosuppressant drug use (defined as "non-HIV immunocompromised patients"). The number and type of histological or microbiological samples which were culture positive for NTM and the number of these samples, which had a positive smear were all recorded. Samples were inoculated onto a solid Lowenstein-Jensen medium. A liquid culture system, mycobacterial growth indication tubes, was not introduced until 1999. The treatment regimens instituted were also analysed along with the success or failure of treatment regimes defined as clinical deterioration associated with failure to eradicate the NTM or recurrence of disease confirmed on culture within 2 yrs of cessation of treatment. In vitro sensitivity tests for individual drugs were performed by the Mycobacterium Reference Units for the UK. The data identifying each individual's NTM sample profile, antibiotic sensitivities and follow-up samples for evidence of recurrence of infection were all extracted for analysis.

In 2001 and 2002 a telephone questionnaire was applied to the same patients or relatives of patients, verified above, to identify potential epidemiological factors which may have contributed to the development of NTM infection, e.g. a weekly use of natural bottled spring water, exposure to pet birds, particularly psitticine birds and pigeons, and a patient's occupation [7, 8]. Data was also collected on a patient's outcome including retreatment or death. For each city ward in Leeds Health Authority, a deprivation score, the Carstairs Index, was calculated using the 2001 census [15]. Populationweighted densities were calculated by ward as this measure was considered to reflect more accurately the density at which the average person lives compared with the area-based population densities.

The diagnostic criteria used to support the treatment of NTM clinical infection in this group of patients as opposed to colonisation of secretions [16] in the absence of clinical disease were compared with subsequently published ATS or BTS diagnostic criteria for NTM. These criteria include the following.

American Thoracic Society. In symptomatic patients with infiltrate, nodular or cavitary disease, or a high resolution computed tomography scan which shows multifocal bronchiectasis and/or multiple small nodules, the following apply: 1) three positive sputum/bronchial wash cultures with negative acid-fast bacillus (AFB) smear or two positive cultures and one positive AFB smear in the last 12 months; 2) if only one bronchial wash available then a positive culture with a $2+$ to $4+$ AFB smear or $2+$ to $4+$ growth on solid media; 3 ) if sputum or bronchial wash are nondiagnostic then a transbronchial or lung biopsy yielding nontuberculous mycobacteria or biopsy showing mycobacterial histopathological features and one or more sputums, or bronchial washings, are positive for nontuberculous mycobacteria even if in low numbers [12].

British Thoracic Society. Pulmonary disease: M. kansasii, $M$. avium intracellulare complex infections, $M$. malmoense, M. xenopi. Pulmonary disease is diagnosed when positive cultures develop from specimens of sputum obtained at least 7 days apart in a patient whose chest radiograph suggests mycobacterial infection and who may or may not present with symptoms and signs [13].
Likewise antibiotic treatment regimens were compared with the BTS management guidelines for NTM infection [12, 13].

\section{Statistical methods}

Much of the data provided is presented as summary statistics. Relative success of treatment regimens in the different NTM subgroups were compared using the Chisquared test and Fisher's exact test, and were considered significant when the p-value was $<0.05$.

\section{Results}

Between 1995-1999, 117 non-HIV positive patients had clinical specimens taken by clinicians in Leeds, which were subsequently found on culture to have a growth of NTM. A total of 71 patients, from the initial 117, were felt by the clinicians involved to have had clinically relevant disease and their decisions, when reviewed retrospectively, were supported by diagnostic criteria laid down by the ATS relating to NTM. Forty-nine patients were diagnosed as suffering from clinically relevant pulmonary disease and 22 patients were felt to have clinically relevant extrapulmonary disease. Table 1 outlines the demographic characteristics of the patients involved.

The incidence of NTM infections increased over this period of time. Figure 1 illustrates the annual incidence of NTM infections between 1995 when $6(0.8$ out of 100,000$)$ clinically relevant infections were diagnosed, to a peak of 22 (3.07 out of 100,000$)$ infections in 1997 and 14 (1.95 out of 100,000$)$

Table 1.-Characteristics of 71 patients suffering from nontuberculous mycobacterial infections in Leeds 1995-1999

\begin{tabular}{|c|c|c|c|c|}
\hline \multirow[t]{2}{*}{ Characteristic } & \multicolumn{3}{|c|}{ Patients $\mathrm{n}$} & \multirow{2}{*}{$\begin{array}{c}\text { Total } \\
\text { patients } \\
\%\end{array}$} \\
\hline & Total & Pulm. & Extrapulm. & \\
\hline Total & 71 & & & \\
\hline Pulm. & 51 & & & 72 \\
\hline Extrapulm. & 20 & & & 28 \\
\hline Lymph node & 14 & & & 20 \\
\hline Skin & 5 & & & 7 \\
\hline Locomotor & 1 & & & 1 \\
\hline White & 69 & & & 96 \\
\hline Non-White & 3 & & & 4 \\
\hline \multicolumn{5}{|l|}{ Year of culture } \\
\hline 1995 & 6 & 6 & 0 & 8 \\
\hline 1996 & 14 & 12 & 2 & 20 \\
\hline 1997 & 22 & 12 & 10 & 31 \\
\hline 1998 & 15 & 9 & 6 & 21 \\
\hline 1999 & 14 & 12 & 2 & 20 \\
\hline \multicolumn{5}{|l|}{$\begin{array}{l}\text { Exposure to } \\
\text { psitticine birds }\end{array}$} \\
\hline Yes & 9 & & & 13 \\
\hline No & 62 & & & 87 \\
\hline \multicolumn{5}{|l|}{$\begin{array}{l}\text { Socio-economic } \\
\text { status }\end{array}$} \\
\hline I & 2 & & & 3 \\
\hline II & 13 & & & 18 \\
\hline III & 22 & & & 31 \\
\hline IV & 22 & & & 31 \\
\hline $\mathrm{V}$ & 12 & & & 17 \\
\hline Immunocompromised & 19 & & & 27 \\
\hline Immunocompetent & 52 & & & 73 \\
\hline
\end{tabular}

Pulm.: pulmonary; Extrapulm.: extrapulmonary. 


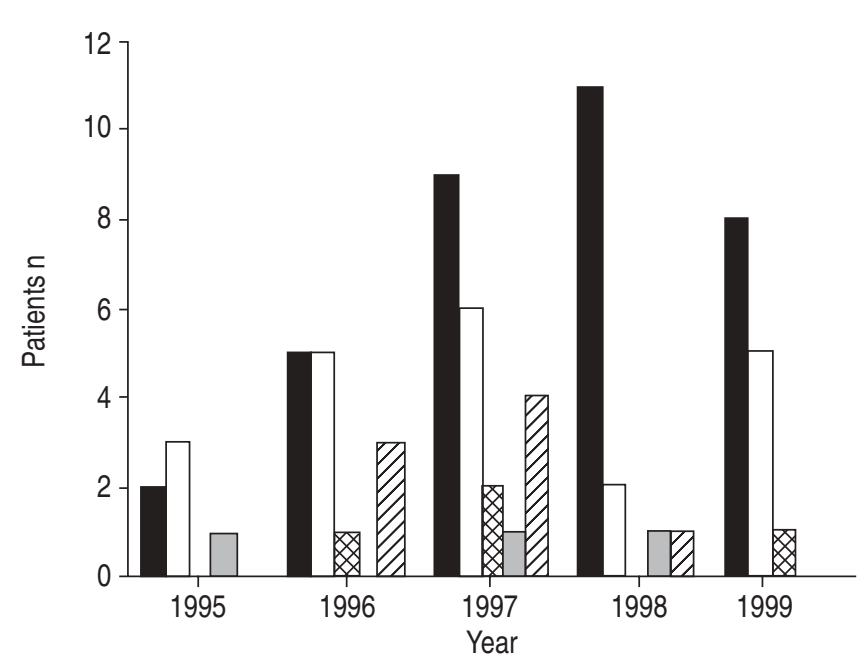

Fig. 1.-Incidence of nontuberculous mycobacterial infections in Leeds 1995-1999 subdivided by subtype and year. 口: Mycobacterium avium intracellulare complex; $\square$ : Mycobacterium malmoense: Mycobacterium kansasii; : Mycobacterium xenopi; $\mathbb{Z}$ : others.

infections in 1999. The mean age of patients in the $3 \mathrm{yrs}$ with lowest incidence, 1995, 1996 and 1999, was 53.4 yrs. This compares with a mean age of 41.6 yrs for 1997 and 1998. There was a notable increase in the proportion of $M$. avium intracellulare complex (MAC) infections over this period of time $(33 \%$ of the total in $1995,36 \%$ in $1996,41 \%$ in $1997,61 \%$ in 1998 and $57 \%$ in 1999). The peak high incidence of MAC disease in 1997 related to five cases $(62.5 \%)$ of MAC lymph node disease in children, whereas in 1998, the high incidence seemed to relate to a peak ( 8 out of $11: 73 \%$ ) in the number of respiratory infections. Other notable increases included a peak in M. marinum skin infections in 1997 (17\%). Otherwise there did not appear to be a trend in the incidence of individual opportunistic infections over this 5-yr period. There was also an increase in incidence in NTM infections as a proportion of total number of recorded mycobacterial infections over this period ( $8 \%$ in $1995,14 \%$ in $1996,18 \%$ in $1997,15 \%$ in 1998 and $14 \%$ in 1999).

Among the 117 patients who had positive cultures during the review period within Leeds, it was possible to map the Carstairs deprivation index of 102 of these cases [15]. A total of $34.4 \%$ of cases lived in the most deprived areas (Carstairs Index 3.9-9.5), 23.5\% in areas with a Carstairs Index of $0.3-3.9,21.5 \%$ in areas with a Carstairs Index of $-2.4-0.3$ and $20.6 \%$ of cases lived in the areas of least deprivation (Carstairs Index -7.2- -2.4). There was no apparent relationship between individual NTM and deprivation index. Subgroup analysis did not identify any difference between pulmonary and extrapulmonary disease as regards antigen exposure or deprivation index.

Fourteen patients received treatment inappropriately for NTM infection. These patients received treatment without fulfilling ATS criteria for diagnosis of clinical infection. From these 14 patients, 10 were treated after a single positive sputum culture and/or a degree of clinical suspicion. Only three of these patients had a sample sent from a sterile site before treatment. A total of $50 \%$ of this group of patients $(n=7)$ died or deteriorated and $50 \%$ improved or recovered. The average length of inappropriate antibiotic therapy was 12 months. In contrast, amongst those patients who were diagnosed and treated with appropriate antibiotics in accordance with the ATS diagnostic criteria, 28 patients had only a single clinical sample sent for culture and 26 of these patients had a diagnosis made on the basis of culture from a sample from a sterile site (predominmantly lymph node biopsies), smear positivity and a strong clinical suspicion including MAC lymph-node disease in children and dermatological disease (M. marinum-fish tank granuloma) [17, 18]. Thirty five per cent of this group died or deteriorated over the following 2 yrs, the remaining $65 \%$ improved or recovered.

A review of predisposing pulmonary conditions in the group of patients with a secure diagnosis of NTM disease showed that $37 \%$ had no underlying lung disease. The pattern of underlying lung disease and species of NTM infection in each disease subtype is outlined in table 2.

There did not appear to be a pattern of species specificity for particular respiratory disease subtypes apart from an apparent increase in $M$. malmoense infections in chronic

Table 2. - Treatment regimens used for nontuberculous mycobacterial pulmonary infections

\begin{tabular}{|c|c|c|c|c|c|c|c|c|c|c|c|}
\hline & \multirow[t]{2}{*}{$\begin{array}{c}\text { Total } \\
\mathrm{n}\end{array}$} & \multicolumn{3}{|c|}{$\begin{array}{c}\text { Treatment } \\
\text { Appropriateness }\end{array}$} & \multicolumn{2}{|c|}{$\begin{array}{l}\text { Died of disease } \\
\text { recurrence }\end{array}$} & \multicolumn{3}{|c|}{$\begin{array}{l}\quad \text { Received } \geqslant 2 \\
\text { antibiotics to which } \\
\text { NTM were sensitive }\end{array}$} & \multicolumn{2}{|c|}{$\begin{array}{l}\text { Died or disease } \\
\text { recurrence }\end{array}$} \\
\hline & & & $\mathrm{n}$ & $\%$ & $\mathrm{n}$ & $\%$ & & $\mathrm{n}$ & $\%$ & $\mathrm{n}$ & $\%$ \\
\hline \multirow[t]{2}{*}{ Mycobacterium avium complex } & 22 & Yes & 13 & 59 & 4 & 31 & Yes & 5 & 23 & 1 & 20 \\
\hline & & No & 9 & 41 & 7 & $\begin{array}{c}78 \\
p=0.08\end{array}$ & No & 17 & 77 & 8 & $\begin{array}{c}53 \\
p=0.3\end{array}$ \\
\hline \multirow[t]{2}{*}{ Mycobacterium malmoense } & 18 & Yes & 8 & 44 & 2 & 25 & Yes & 9 & 50 & 3 & 33 \\
\hline & & No & 10 & 56 & 8 & $\begin{array}{c}80 \\
\mathrm{p}=0.05\end{array}$ & No & 9 & 50 & 6 & $\begin{array}{c}66 \\
p=0.35\end{array}$ \\
\hline \multirow[t]{2}{*}{ Mycobacterium Kansasii } & 3 & Yes & 1 & 33 & 0 & 0 & Yes & 2 & 67 & 0 & 0 \\
\hline & & No & 2 & 66 & 1 & 50 & No & 1 & 33 & 1 & 100 \\
\hline \multirow[t]{2}{*}{ Mycobacterium xenopi } & 3 & Yes & 1 & 33 & 0 & 0 & Yes & 1 & 33 & 0 & 0 \\
\hline & & No & 2 & 66 & 1 & 50 & No & 2 & 67 & 1 & 50 \\
\hline Others & & Yes & 0 & 0 & 0 & 0 & Yes & 0 & 0 & 0 & 0 \\
\hline Rapidly growing Mycobacterium & 3 & No & 3 & 100 & 2 & 66 & No & 3 & 100 & 2 & 67 \\
\hline \multirow[t]{2}{*}{ Total } & 49 & Yes & 23 & 47 & 6 & 24 & Yes & 17 & 35 & 4 & 23 \\
\hline & & No & 26 & 53 & 19 & $\begin{array}{c}76 \\
p=0.001\end{array}$ & No & 32 & 65 & 19 & $\begin{array}{c}59 \\
\mathrm{p}=0.03\end{array}$ \\
\hline
\end{tabular}

The appropriateness of treatment was defined using American Thoracic Soiciety and British Thoracic Society criteria [12, 13]. Death or disease recurrence occurred within 2 yrs of treatment cessation. NTM: nontuberculous mycobacteria. 


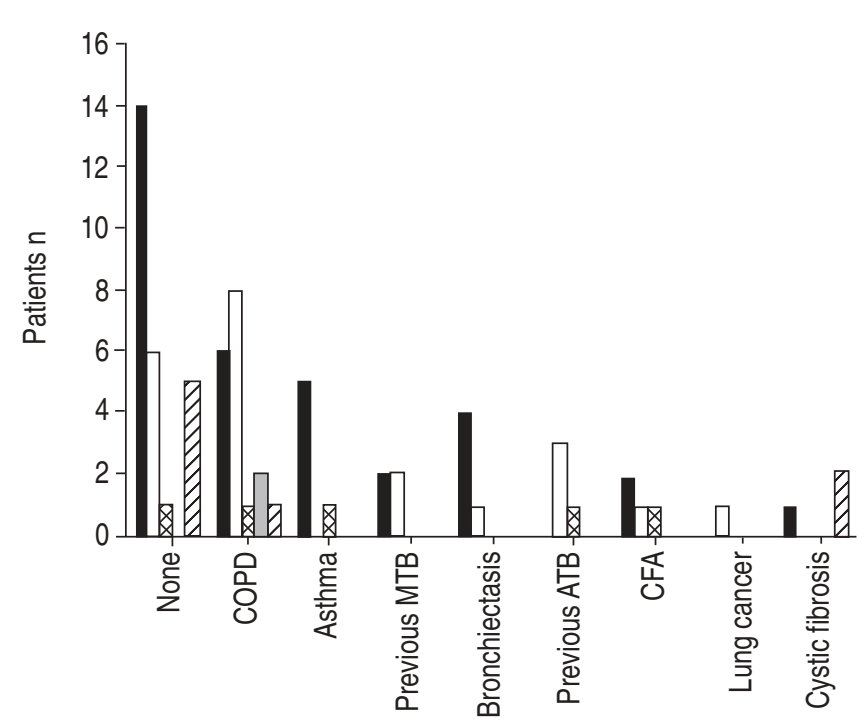

Fig. 2. - Underlying lung disease in patients subgrouped according to individual nontuberculous mycobacteria. - : Mycobacterium avium intracellulare complex; $\square$ : Mycobacterium malmoense: $\mathbf{a y c o b a c t e r -}$ ium kansasii; $\square$ : Mycobacterium xenopi; $\mathbb{Z}$ : others. COPD: chronic obstructive pulmonary disease; MTB: Mycobacterium tuberculosis; ATB: atypical mycobacteria; CFA: cryoptogenic fibrosing alveolitis.

obstructive pulmonary disease (COPD) patients and, not surprisingly, a lack of any underlying lung disease in $M$. marinum disease patients. The mortality rate among COPD patients $(n=20)$ suffering from NTM infections was $55 \%$, among bronchiectasis patients $(n=6)$ was $66 \%$ and among cystic fibrosis patients $(n=3)$ was $100 \%$ within 2 yrs of diagnosis, perhaps reflecting severity of the underlying condition rather than an independent association with NTM infection. In contrast the mortality among asthma patients with NTM infections $(n=6)$ was $0 \%$ within 2 yrs of diagnosis.

The BTS has outlined specific antibiotic regimens, which should be used, and the duration of treatment for first and recurrent pulmonary NTM infections. These recommendations are based on the best available evidence $[3,12,13$, 19-23]. It has been suggested that success of treatment may be related to the adherence to correct antibiotic regimens [24, 25]. Suggested treatments for pulmonary nontuberculous mycobacterial pathogens include the following.

M. avium complex, M. malmoense, M. xenopi, pulmonary disease

Rifampicin+ethambutol \pm isoniazid \pm clarithromycin or ciprofloxacin. Treatment for 2 yrs. If recurrent disease treat indefinitely or until sputum clear for 12 months.

\section{M. kansasii pulmonary disease}

Rifampicin+ethambutol \pm isoniazid \pm clarithromycin or ciprofloxacin. Treatment for 9 months. If recurrent disease treat indefinitely or until sputum clear for 12 months.

\section{Pulmonary disease due to rapidly growing mycobacteria (e.g. M. chelonae, M. szulgai)}

Rifampicin+ethambutol+clarithromycin. Treatment time not specified, probably indefinite [12].

The 49 patients with clinically significant NTM pulmonary disease during this period were divided into those who received appropriate antibiotic treatment regimens and those who received inappropriate regimens as defined above. They either received the wrong combination of antibiotics and/or treatment for an inappropriately short period of time or both. Table 2 outlines the treatment regimens given for these patients. The striking feature of the treatment that these patients received was that $53 \%$ of these patients were treated using antibiotic regimens that would now be considered suboptimal or inappropriate. The rate of disease recurrence and/or mortality amongst the patients who received inappropriate treatment was $76 \%$ and significantly higher than those who received appropriate therapy (ranging from $50 \%$ amongst $M$. kansasii patients to $80 \%$ amongst $M$. malmoense patients). These figures compare strikingly with a recurrence and/or death rate of $26 \%$ among patients who received effective and appropriate therapeutic antibiotic regimens. The odds ratio for recovery from NTM pulmonary disease was 7.7 (95\% CI: 2.1-27.4) among those who received appropriate therapy compared to those who did not. During the study and follow-up period, there were 19 patients who received inappropriate treatment who had an unsuccessful outcome. Eighteen of these patients received incorrect antibiotics according to the regimens outlined above. All 19 patients were treated for too short a period of time and 11 patients received both incorrect antibiotics and were treated too briefly. This group of patients was further subdivided into those who were treated from the outset with at least two antibiotics to which they were subsequently shown to be sensitive and those who were not. Table 2 demonstrates a significantly better outcome in patients who received at least two antibiotics to which their NTM isolate was sensitive. The odds ratio of a favourable outcome in this group was 4.7 (95\% CI: $1.3-17.8)$.

In general, standard in vitro drug sensitivity of NTM isolates is of little use in predicting clinical efficacy particularly in those receiving repeat treatment [26]. The single exception to this is in vitro sensitivity of $M$. kansasii to rifampicin and ethambutol. Likewise, synergy between antibiotics in vivo maybe important in cases where resistance to the individual antibiotics has been reported [21,27]. Susceptibility

Table 3. - In vitro antibiotic sensitivities received from UK Mycobacterium Reference Units for the individual nontuberculous mycobacteria isolated in Leeds between 1995-1999

\begin{tabular}{lcccccc}
\hline Isolates sensitive \% & Rifampicin & Rifabutin & Isoniazid & Ethambutol & Clarithromycin/azithromycin & Ciprofloxacin \\
\hline Mycobacterium avium complex & 11 & 13 & 0 & 20 & 93 & 7 \\
Mycobacterium malmoense & 32 & 62 & 0 & 61.5 & 75 & 100 \\
Mycobacterium kansasii & 82 & 82 & 0 & 33 & 100 & 12 \\
Mycobacterium xenopi & 67 & 0 & 0 & 80 & 32 & 100 \\
Mycobacterium marinum & 80 & 75 & 0 & 33 & 0 \\
Others & 0 & 33 & 0 & & 33 \\
\hline
\end{tabular}

\#: $\mathrm{n}=3$. 
testing of the MAC for first-line antituberculousis drugs is not recommended but there may occasionally be a case for testing against clarithromycin. [12, 13, 21, 26-28]. All clinically significant isolates of $M$. kansasii should be tested against rifampicin. In the case of rifampicin resistance, sensitivities to isoniazid, ethambutol, clarithromycin and ciprofloxacin may be important [12, 28]. Antibiotic sensitivity of all rapidly growing isolates should be available [12, 28].

Table 3 outlines the antibiotic sensitivities for all 117 NTM isolates in Leeds in 1995-1999. It is interesting that none of the isolates were sensitive to isoniazid. A recent study from the BTS Research Committee comparing rifampacin and ethambutol with rifampicin, ethambutol and isoniazid for the treatment of $M$. malmoense patients concluded that better regimens incorporating rifampicin, ethabutol and other second line antituberculous drugs are needed [23]. There are ongoing studies comparing clarithromycin or ciprofloxacin in addition to rifampicin and ethambutol in $M$. avium complex or M. malmoense pulmonary infections. Notwithstanding the fact that there is, as yet, no clinical in vitro evidence to support the use of one over the other, table 3 shows a clear superiority of clarithromycin (or azithromycin) over ciprofloxacin in in vitro sensitivity testing in the samples tested.

\section{Discussion}

The incidence of NTM infections has increased particularly among immunosuppressed patients. However, as this study demonstrates, there may also be a rising incidence of nonimmunosuppressed persons with clinical NTM disease. While one could speculate that this rise may reflect increased surveillance for NTM infection amongst patients with chronic respiratory disease, particularly after 1997 when the first definitive investigation and treatment guidelines were published [12], during the period of this review the authors were unable to identify any increase in screening for NTM infection amongst either the pulmonary physicians, paediatricians, surgeons or any other group involved in the treatment of these patients. In particular there were no new consultant appointments with an interest in this area and there was no change in policy for tuberculosis (TB) screening or culture methods in Leeds during this time. There was no increase in number of biological samples tested for TB or NTM infection over this period. Neither was there any policy for increased surveillance for NTM disease amongst pulmonary radiologists, microbiologists or public health doctors during this period. The peak in 1997 is likely to have been related to a higher incidence of MAC lymph-node disease in children than usual. The peak of MAC infection in 1998 does appear to relate to a peak in respiratory infections that year. Higher incidences of clinical disease related to NTM and in particular MAC over these 2 yrs have been reported in other series in similar climates and populations [32], and in UK in communicable disease reports over that period.

The majority of pulmonary NTM disease occurred in patients with underlying lung disease in this study. However, $41 \%$ of patients in this study who developed significant NTM pulmonary infections had no underlying lung disease. MAC infections in non-HIV patients without pre-existing lung disease have been described in the literature in recent years with increasing frequency $[4,9,22,29]$. In this study, $66 \%$ of patients with pulmonary NTM infection without underlying lung disease suffered MAC pulmonary infections. This group also accounts for $20 \%$ of all clinical NTM infections between 1995-1999. This is similar to the $18 \%$ of 119 patients described by PRINCE et al. [9] as having MAC pulmonary infection without predisposing conditions. The clinical implications of this finding as described previously are that these patients have a long delay before diagnosis is made and a high recurrence rate.

The overall incidence of NTM infections and in particular MAC infections increased in Leeds between 1995 (0.8 out of 100,000) and $1999(1.95$ out of 100,000) with a peak in 1997 (3.07 out of 100,000$)$. This is considerably higher than a comparable report $(0.62$ out of 100,000$)$ in a predominantly $(>99 \%)$ indigenous Caucasian population in southwest Ireland, an area of similar climate, recently reported, although this group did report a steady rise in NTM infections in a HIV-negative population. They also reported a peak in infections in 1997, which were predominantly MAC infections [30]. The present figures are similar to those published in north Australia (3.9 out of 100,000$)$ and the USA (2.1 out of $100,000)$ areas with a more mixed racial population similar to that of Leeds [31, 32]. The rise in NTM infections in the present study population is proportionally larger than the rise in cases of TB over the same period of time.

There is an established geographical variation in nontuberculous mycobacterial infections throughout the UK with M. Kansasii infection predominating generally in England and Wales, M. malmoense infections in Scotland, and $M$. xenopi in the south east of England [11]. MAC has been reported as accounting for up to $60 \%$ of isolates in the USA [33]. MAC accounted for $48 \%$ of NTM infections in non-HIV patients in Leeds. No epidemiological link was established with the high incidence of MAC infections in Leeds. While no link was established between race, exposure to psitticine birds, sex or immune status in HIV-negative patients and the incidence of opportunistic infections, there was a clear link between lower socio-economic status and NTM infection in this study. This mirrors previous studies, which demonstrated a greater incidence of tuberculosis independent of race in the more deprived areas of the city [34].

The BTS have provided evidence-based recommendations for the treatment of pulmonary and extra-pulmonary NTM infections [13]. Prior to the publication of evidence-based guidelines in 1997 and 1999, there was a lack of consensus reflecting an absence of large clinical trials and as a result treatment of such infections was less regimented, as evidenced by the number of different regimes used to treat pulmonary infections in this study. While this is a retrospective review of treatment, it is felt that the data presented strongly support an association between correct evidence based regimens and outcome. The physicians treating patients described in this report did so prior to the publication and dissemination of the ATS and BTS guidelines. While $53 \%$ of the treatment regimens used between 1995-1999 would now be considered inappropriate, $76 \%$ of these patients suffered recurrence of disease or died within 2 yrs of cessation of treatment. In comparison only $24 \%$ of patients who were treated using regimens subsequently supported by evidence-based BTS guidelines died or suffered disease recurrence within the same period. The authors feel that this evidence supports the appropriateness of the BTS guidelines for the treatment of NTM in non-HIV patients [13].

The BTS and ATS guidelines for pulmonary NTM treatment provide evidence-based guidance on treatment of NTM infections. The best regimens available, used in clinical trial settings, would suggest that $90 \%$ clinical response rates for M. kansasii treatment, $70 \%$ for MAC, $90 \%$ for M. malmoense and $45 \%$ for $M$. xenopi pulmonary infections can be achieved by adhering to evidence regimens [2-6, 19-21, 23]. In this reterospective review, carried out in a less controlled clinical trial setting, MAC pulmonary infections treated with appropriate regimens achieved a successful outcome in $69 \%$ of cases and $M$. malmoense in $75 \%$ of cases. In vitro sensitivities suggests that the addition of clarithromycin or azithromycin to these regimens either in 
addition or in place of isoniazid is liable to be more successful than the addition of ciprofloxacin, which has been suggested as a possible alternative in the BTS guidelines. In this study between $7-42 \%$ of MAC and $M$. malmoense isolates were sensitive to ciprofloxacin as opposed to between $87-100 \%$ of isolates which were sensitive to clarithromycin/azithromycin. The numbers of $M$. kansasii and $M$. xenopi treated with appropriate regimens are too small to make valid comments on.

In summary this survey provides descriptive epidemiology of a series of conditions that continue to be a significant problem in the UK. The results support a link with lower socio-economic class and nontuberculous mycobacteria disease. The importance of adherence with recently published evidence based guidelines for the treatment of nontuberculous mycobacteria pulmonary disease is highlighted by the marked contrast in outcomes between those patients who received appropriate treatment regimens and those who did not. Continued vigilance amongst populations at risk and strict adherence to published international treatment guidelines should improve outcomes in these conditions.

\section{References}

1. Marks J, Jenkins PA. The opportunist mycobacteria -20 year reterospect. Postgrad Med J 1971; 47: 705-709.

2. Banks J, Hunter A, Campbell IA, Smith AP. Pulmonary infection with Mycobacterium kansasii in Wales 1970-1979: a review of treatment and response. Thorax 1983; 38: 271-274.

3. Hunter AM, Campbell IA, Jenkins PA, Smith AP. Treatment of pulmonary infections caused by the Mycobacterium avium intracellulare complex. Thorax 1981; 36: 326-329.

4. Huang JH, Kao PN, Adi V, Ruoss SJ. Mycobacterium avium intracellulare pulmonary infections in HIV-negative patients without pre-existing lung disease. Chest 1999; 115: 1033-1040.

5. Henriques B, Hoffner SE, Petrini B, Juhlin I, Wahlen P, Kallenius G. Infection of Mycobacterium malmoense in Sweden: Report of 221 cases. Clin Infect Dis 1994; 18: 596-600.

6. Banks J, Hunter AM, Campbell IA, Jenkins PA, Smith AP. Pulmonary infection with Mycobacterium xenopi. Review of treatment and response. Thorax 1984; 39: 376-382.

7. Marras TK, Daley CL. Epidemiology of human pulmonary infection with nontuberculous mycobacteria. Clin Chest Med 2002; 23: 553-568.

8. McGarvey J, Bermudez LE. Pathogenesis of nontuberculous mycobacteria infection. Clin Chest Med 2002; 23: 569-584.

9. Prince DS, Peterson DD, Steiner RM, et al. Infection with Mycobacterium avium Complex in patients without predisposing conditions. N Engl J Med 1989; 321: 863-868.

10. $\mathrm{O}^{\prime}$ Brien RJ, Geiter LJ, Snider DE Jr. The epidemiology of nontuberculous mycobacterial diseases in the United States: results from a national survey. Am Rev Respir Dis 1987; 135: 1007-1014.

11. Lambden K, Watson JM, Knerer G, Ryan MJ, Jenkins PA. Opportunist mycobacteria in England and Wales 1982-1994. CDR Review 1996; 11: 147-151.

12. American Thoracic Society. Wallace RJ Jr, Glassroth J, et al.. American Thoracic Society. Diagnosis and treatment of disease caused by nontuberculous mycobacteria. Am J Respir Crit Care Med 1997; 156: S1-S25.

13. Subcommittee of the Joint Tuberculosis Committee of the British Thoracic Society. Management of opportunist mycobacterial infections: Joint Tuberculosis Committee guidelines 1999. Thorax 2000; 55: 210-218.

14. 2001 UK Census: Office for Population, Censuses and Surveys. www.statistics.gov.uk/census2001/profiles/ooda.asp\# ethnic. Date last updated: September 2003. Date accessed: December 2003

15. Carstairs V, Morris R. Deprivation and health in Scotland. Aberdeen, Aberdeen University Press, 1991.

16. Catanzaro A. Diagnosis, differentiating colonisation, infection and disease. Clin Chest Med 2002; 23: 599-602.

17. Wolinski E. Mycobacterial lymphadenitis in children: a prospective study of 105 nontuberculous cases with long term follow up. Clin Infect Dis 1995; 20: 954-963.

18. Travis WD, Travis LB, Roberts GD, Su DW, Weiland LW. The histopatological spectrum in Mycobacterium marinum infection. Arch Pathol Lab Med 1985; 109: 1109-1113.

19. British Thoracic Society. Mycobacterium kansasii pulmonary infection: a prospective study of the results of nine months of treatment of rifampicin and ethambutol. Thorax 1994; 49: 442-445.

20. British Thoracic Society. First randomised trial of treatments for pulmonary disease caused by $M$. avium intracellulare, $M$. malmoense and $M$. xenopi in HIV-negative patients: rifampicin, ethambutol and isoniazid versus rifampicin and ethambutol. Thorax 2001; 56: 167-172.

21. Banks J, Jenkins PA. Combined versus single antituberculosis drugs on the in vitro sensitivity patterns of nontuberculous mycobacteria. Thorax 1987; 42: 838-842.

22. Reich JM, Johnson RE. Mycobactereium avium complex pulmonary disease. Incidence, presentation, and response to therapy in a community setting. Am Rev Respir Disease 1991; 143: $1381-1385$

23. British Thoraacic Society. Pulmonary disease caused by $M$. malmoense in HIV negative patients. 5-year follow-up of patients receiving standardised treatment. Eur Respir $J$ 2003; 21: $478-482$

24. Askamit TR. Mycobacterium avium complex pulmonary disease in patients with pre-exisiting lung disease. Clin Chest Med 2002; 23: 643-654.

25. Griffith DE. Management of disease due to Mycobacterium kansasii. Clin Chest Med 2002; 23: 613-622.

26. Heifets LB. Susceptibly testing for Mycobacterium avium isolates. Antimicrob Agents Chemother 1996; 40: 1759-1767.

27. Heifets LB. Synergistic effect of rifampicin, streptomycin, ethionamide and ethambutamol on Mycobacterium intracellulare. Am Rev Respir Dis 1982; 125: 43-48.

28. National Committee for Clinical Laboratory Standards. Susceptibility testing for Mycobacteria, Nocardia and other anaerobic Actinomycetes. Tentative standard 2nd Edn. NCCLS document M24T2. Wayne, PA, National committee for clinical laboratory standards, 2000.

29. Iseman MD. Mycobacterium avium complex and the normal host: the other side of the coin. N Engl J Med 1989; 321: 896898.

30. Kennedy MP, O'Connor TM, Ryan C, Sheehan S, Cryan B, Bredin C. Nontuberculous mycobacteria: incidence in Southwest Ireland from 1987 to 2000. Respir Med 2003; 97: 257-263.

31. O'Brien DP, Currie BJ, Krause VL. Nontuberculous mycobacterial disease in northern Australia: case series and review of the literature. Clin Infect Dis 2000; 31: 958-967.

32. O'Brien RJ, Geiter LJ, Snider DE. The epidemiology of nontuberculous mycobacterial diseases in the United States: results from a national survey. Am Rev Respir Dis 1987; 135: $1017-1024$

33. Good RC, Snider DE. Isolation of non-tuberculous mycobacteria in the United States, 1980. J Infect Dis 1980; 146: 829-833.

34. Goldman JM, Teale C, Cundall DB, Pearson SB. Childhood tuberculosis in Leeds, 1982-1990: social and ethnic factors and the role of the contact clinic in diagnosis. Thorax 1994; 49: $184-185$ 